\title{
Density functional theory of electrowetting
}

\author{
Markus Bier* and Ingrid Ibagon \\ Max-Planck-Institut für Intelligente Systeme, Heisenbergstr. 3, \\ 70569 Stuttgart, Germany, and Institut für Theoretische Physik IV, \\ Universität Stuttgart, Pfaffenwaldring 57, 70569 Stuttgart, Germany
}

(Dated: 10 February 2014)

\begin{abstract}
The phenomenon of electrowetting, i.e., the dependence of the macroscopic contact angle of a fluid on the electrostatic potential of the substrate, is analyzed in terms of the density functional theory of wetting. It is shown that electrowetting is not an electrocapillarity effect, i.e., it cannot be consistently understood in terms of the variation of the substrate-fluid interfacial tension with the electrostatic substrate potential, but it is related to the depth of the effective interface potential. The key feature, which has been overlooked so far and which occurs naturally in the density functional approach is the structural change of a fluid if it is brought into contact with another fluid. These structural changes occur in the present context as the formation of finite films of one fluid phase in between the substrate and the bulk of the other fluid phase. The non-vanishing Donnan potentials (Galvani potential differences) across such film-bulk fluid interfaces, which generically occur due to an unequal partitioning of ions as a result of differences of solubility contrasts, lead to correction terms in the electrowetting equation, which become relevant for sufficiently small substrate potentials. Whereas the present density functional approach confirms the commonly used electrocapillarity-based electrowetting equation as a good approximation for the cases of metallic electrodes or electrodes coated with a hydrophobic dielectric in contact with an electrolyte solution and an ion-free oil, a significantly reduced tendency for electrowetting is predicted for electrodes coated with a dielectric which is hydrophilic or which is in contact with two immiscible electrolyte solutions.
\end{abstract}

\section{INTRODUCTION}

Since the pioneering work of Lippmann [1] and Pellat [2, 3] on the influence of electrostatic potentials on the wetting of substrates by fluids, electrowetting has been simultaneously studied to address fundamental issues of surface science, e.g., electrocapillarity [4], the structure of solid-fluid interfaces [5], or the characterization of surface states [6], as well as to develop novel applications, e.g., driving, mixing, or shaping of droplets in lab-on-a-chip devices, optical applications, or microelectromechanical systems [7]. In the past electrowetting at low voltages was commonly interpreted as an electrocapillarity effect, i.e., it is assumed to hinge on the voltage-dependence of the substrate-fluid interfacial tension [2, [3, 5, 7] 19]. A justification for this approach is frequently given in terms of the vast experimental evidence for systems of uncoated and hydrophobically coated electrodes.

The present work reports on an effort to understand electrowetting in the context of general wetting phenomena [20]. Within classical microscopic density functional theory one has access to the interfacial structure of fluids so that one can study, e.g., the contact angle as a function of the electrostatic substrate potential. However, it turned out that the commonly given derivations of the electrowetting equation [7] are incorrect in that interfacial properties, e.g., interfacial tensions or differential capacitances, which describe the contact of one fluid with

\footnotetext{
*Electronic address: bier@is.mpg.de

${ }^{\dagger}$ Electronic address: ingrid@is.mpg.de
}

a substrate, enter the equation describing the contact of two fluids with a substrate. It has been overlooked that the interfacial structure, and thus interfacial quantities, of a fluid can change upon bringing it into contact with another fluid. Although the interfacial structure close of fluids to substrates has been deeply examined in the surface science literature 21 27], its properties seem to be largely ignored in the context of electrowetting so far. By ignoring structural differences which occur at substrate-fluid interfaces upon bringing two fluids in simultaneous contact with a substrate, one can interpret electrowetting as a consequence of voltage-dependent interfacial tensions, which is referred to as the electrocapillarity approach to electrowetting in the following. It is shown in this work that electrowetting cannot be consistently understood as an electrocapillarity effect. Alternative approaches to interpret electrowetting as a line tension effect have been proposed [28] but some of the predictions were in disagreement with experimental data [29].

The present approach is to study electrowetting in terms of the the effective interface potential [20], which is related to the macroscopic contact angle. The effective interface potential has been analyzed recently for simple models of electrolyte solutions [30 32], showing the general feature of ions inducing wetting transitions of first order. Whereas wetting transitions are concerned with the thickness of wetting films, the contact angle is related to the depth of the effective interface potential, which always vanishes continuously at wetting transitions [20]. In the following the effective interface potential for Pellat's classical setup of a vertical parallel plate capacitor [2, 3] is determined and used to derive an electrowetting equation 


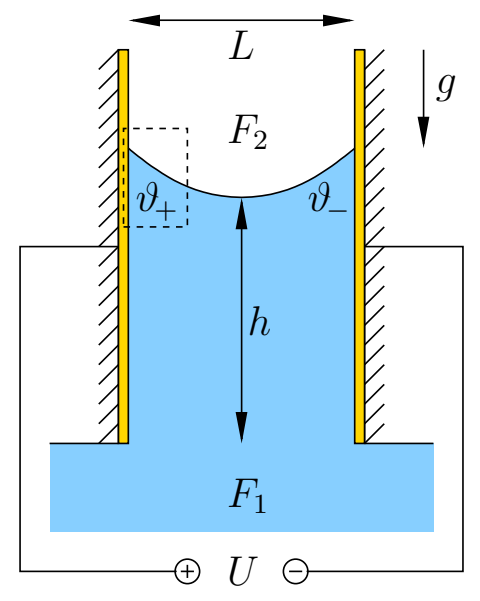

FIG. 1: Pellat's setup [2, 3] of electrocapillary rise of a fluid $F_{1}$ in a vertical parallel plate capacitor of width $L$ initially filled with a fluid $F_{2}$. The meniscus height $h$ is related to the contact angles $\vartheta_{+}$and $\vartheta_{-}$by Eq. (2). Electrowetting corresponds to a dependence of $\vartheta_{+}$and $\vartheta_{-}$, and hence of $h$, on the voltage $U$ between the plate electrodes. A closeup of the three-phase contact region marked by the dashed box is depicted in Fig. 2

(Sec. III) based on the density functional theory of wetting. This setup has been chosen because its geometry is precisely defined, an issue which has been recently raised in a critical discussion of the more common setup of a droplet on a substrate with the counter electrode being a thin wire [17]. The misconception underlying the classical derivation of the electrowetting equation within the electrocapillarity approach is discussed (Sec. IIII). Interestingly, the electrocapillarity-based electrowetting equation seems to be a good approximation for systems investigated up to now, i.e., uncoated or hydrophobically coated electrodes (Sec. III). However, it is argued that the difference in the predicted electrowetting numbers between the electrocapillarity approach and the present one based on the density function theory of wetting can be expected to occur, e.g., for hydrophilically coated electrodes or for two immiscible electrolyte solutions as fluids (Sec. III). In view of the conceptional problems of the electrocapillarity approach it is suggested to rather interpret electrowetting in terms of the density functional theory of wetting (Sec. IV). Moreover, the possibility to obtain microscopic information about solid-fluid interfaces by analyzing electrowetting measurements in terms of the density functional theory of wetting deserves further consideration.

\section{THEORETICAL CONSIDERATIONS}

\section{A. Setting}

Consider Pellat's classical setup [2, 3] depicted in Fig. 1. A vertical parallel plate capacitor of width $L$ is in contact with two immiscible fluids $F_{1}$ and $F_{2}$ of mass densities $\varrho_{\mathrm{m} 1}$ and $\varrho_{\mathrm{m} 2}$, respectively. At least one of the fluids $F_{1}$ and $F_{2}$ is assumed to be an electrolyte solution. It is further assumed that $\varrho_{\mathrm{m} 1}>\varrho_{\mathrm{m} 2}$ so that both fluids are separated in the gravitational field with $F_{1}$ being the lower and $F_{2}$ being the upper phase (see Fig. 11). Provided the capacitor width $L$ is smaller than the capillary length 33 33

$$
\lambda=\sqrt{\frac{\gamma_{12}}{\left(\varrho_{\mathrm{m} 1}-\varrho_{\mathrm{m} 2}\right) g}}
$$

with the $F_{1}-F_{2}$ interfacial tension $\gamma_{12}$ and the acceleration due to gravity $g$, the contact angles $\vartheta_{+}$and $\vartheta_{-}$of phase $F_{1}$ are related to the meniscus height $h$ by [33 35$]$

$$
\cos \vartheta_{+}+\cos \vartheta_{-} \simeq \frac{h L}{\lambda^{2}} \quad \text { for } L \ll \lambda .
$$

Depending on the interactions of the fluids $F_{1}, F_{2}$ and the substrates $S_{+}, S_{-}$, which are metal electrodes (represented by the hatched parts in Fig. 1) possibly coated with some dielectric (represented by yellow layers on top of the electrodes in Fig. 11), the respective contact angles $\vartheta_{+}$and $\vartheta_{-}$can be smaller or larger than $\pi / 2$, which corresponds to positive or negative contributions to the meniscus height $h$. Electrowetting can be detected as the dependence of the contact angles $\vartheta_{+}(U)$ and $\vartheta_{-}(U)$, and in turn, via Eq. (2), of the meniscus height $h(U)$, on the electrostatic potential difference $U=\Psi_{+}-\Psi_{-}$applied between the electrodes.

\section{B. Contact angle and effective interface potential}

The contact angles $\vartheta_{+}$and $\vartheta_{-}$in Fig. 1 provide a macroscopic description of the fluid-fluid-substrate threephase contact region (highlighted by the dashed box in Fig. 1 for the anodic substrate $S_{+}$). According to the chemical properties of the fluids and the substrates, the contact of substrate $S_{ \pm}$with one fluid, henceforth denoted by fluid $A$, is more preferable than with the other fluid, henceforth denoted by fluid $B$. Here it is assumed that both substrates are chemically equal such that either fluid $F_{1}$ or fluid $F_{2}$ is preferred by both substrates $S_{+}$and $S_{-}$. Consequently, if substrate $S_{ \pm}$is macroscopically in contact with the bulk of the less preferred fluid $B$ and if the thermodynamic state is away from wetting transitions such that the substrate is only partially wet by phase $A$, a film of microscopic extension $\ell_{ \pm}>\xi$ composed of the preferred fluid $A$ forms in between substrate $S_{ \pm}$and the bulk of fluid $B$ [20], where $\xi$ denotes the bulk correlation length, which is of the order of the particle size if the thermodynamic state is away from critical points. Then the fluid structure is similar to that of a composition of an $S_{ \pm}-A$ interface at the substrate surface and a free $A-B$ interface at a distance $\ell_{ \pm}$away from the substrate, both being of typical extension $\xi$ [20]. This structure leads to a surface contribution 


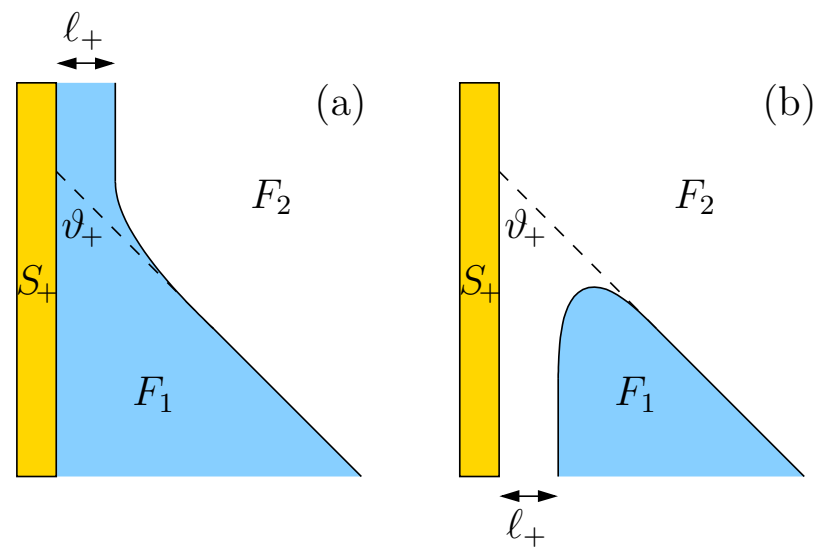

FIG. 2: Closeup of the possible geometries of the three-phase contact region formed by the anodic substrate $S_{+}$(and similarly for the the cathodic substrate $S_{-}$) and two immiscible fluids $F_{1}$ and $F_{2}$ marked by the dashed box in Fig. 11. The fluid which is preferred by the substrates $S_{ \pm}$is denoted by $A$, whereas the other, less preferred fluid is called $B$. Panel (a) corresponds to the case of an $F_{1}$-philic $\left(A=F_{1}\right)$ substrate $S_{+}$, while panel (b) displays the case of an $F_{2}$-philic $\left(A=F_{2}\right)$ substrate $S_{+}$. Here it is assumed that both substrates $S_{+}$ and $S_{-}$prefer the same fluid. The macroscopic contact angle $\vartheta_{+}\left(\vartheta_{-}\right)$shown in Fig. 1 describes the asymptotic inclination of the $F_{1}-F_{2}$ interface far away from the substrate $S_{+}\left(S_{-}\right)$, whereas close to the substrate $S_{+}\left(S_{-}\right)$a film of microscopic thickness $\ell_{+}\left(\ell_{-}\right)$of the preferred fluid $A$ is formed.

$\Omega_{s, \pm B}\left(\ell_{ \pm}\right)=\gamma_{ \pm A}+\gamma_{12}+\omega_{ \pm}\left(\ell_{ \pm}\right)$to the grand potential of the system, where $\gamma_{ \pm A}$ and $\gamma_{12}$ denote the $S_{ \pm}-A$ and $F_{1}-F_{2}$ interfacial tensions, respectively, and where $\omega_{ \pm}\left(\ell_{ \pm}\right)$is the effective interface potential [20]. It is important to distinguish $\Omega_{s, \pm B}$ from the interfacial tension $\gamma_{ \pm B}$ of an $S_{ \pm-} B$ interface in the absence of phase $A$. Here $\Omega_{s, \pm B} \neq \gamma_{ \pm B}$ because the presence of the preferred phase $A$ leads to a structural change, i.e., the formation of $A$-films, as compared to the situation in the absence of phase $A$. Ignoring the difference between $\Omega_{s, \pm B}$ and $\gamma_{ \pm B}$ is equivalent to ignoring the formation of $A$-films and it is this crucial misconception which underlies the electrocapillarity approach to electrowetting. In contrast, if substrate $S_{ \pm}$is in contact with the bulk of the preferred fluid $A$, the fluid is non-uniform only close to the substrate surfaces up to distances $\xi$, and this interfacial structure is not modified by the presence of fluid $B$, hence $\Omega_{s, \pm A}=\gamma_{ \pm A}$. Depending on whether the preferred fluid $A$ is fluid $F_{1}$ or fluid $F_{2}$ the substrates $S_{ \pm}$are referred to as $F_{1}$-philic or $F_{2}$-philic, respectively. A closeup of the fluid-fluid-substrate three-phase contact region close to substrate $S_{+}$marked by the dashed box in Fig. 1] is sketched respectively in Figs. 2(a) and (b) for an $F_{1}$-philic $\left(A=F_{1}\right)$ and an $F_{2}$-philic $\left(A=F_{2}\right)$ substrate.

The macroscopic contact angle $\vartheta_{ \pm}$(see Fig. 2) is related to the surface contributions $\Omega s, \pm 1, \Omega_{s, \pm 2}$ and the interfacial tension $\gamma_{12}$ of the $S_{ \pm}-F_{1}, S_{ \pm}-F_{2}$, and $F_{1}-F_{2}$ interface, respectively, by Young's equation [33 35.

$$
\Omega_{s, \pm 2}=\Omega_{s, \pm 1}+\gamma_{12} \cos \vartheta_{ \pm}
$$

It is common to assume $\Omega_{s, \pm \alpha}=\gamma_{s, \pm \alpha}, \alpha \in\left\{F_{1}, F_{2}\right\}$, but this misconception to ignore the structural differences of a macroscopic $S_{ \pm}-\alpha$ contact in the presence and in the absence of additional phases can have significant consequences. The surface contributions $\Omega_{s, \pm 1}$ and $\Omega_{s, \pm 2}$ are related to the depth of the effective interface potential $\omega_{+}(\ell)$ evaluated at the equilibrium film thickness $\ell=\ell_{ \pm}$ by [20]

$$
\begin{aligned}
& \Omega_{s, \pm 1}=\gamma_{ \pm 1} \\
& \Omega_{s, \pm 2}=\gamma_{ \pm 1}+\gamma_{12}+\omega_{ \pm}\left(\ell_{ \pm}\right)
\end{aligned}
$$

for $F_{1}$-philic substrates $S_{ \pm}$(see above the three-phase contact region in Fig. 2(a)) and by

$$
\begin{aligned}
& \Omega_{s, \pm 1}=\Omega_{s, \pm 2}+\gamma_{12}+\omega_{ \pm}\left(\ell_{ \pm}\right) \\
& \Omega_{s, \pm 2}=\gamma_{s, \pm 2}
\end{aligned}
$$

for $F_{2}$-philic substrates $S_{ \pm}$(see below the three-phase contact region in Fig. 2(b)). Hence, one obtains [20]

$$
\cos \vartheta_{ \pm}=\frac{\Omega_{s, \pm 2}-\Omega_{s, \pm 1}}{\gamma_{12}}=p\left(1+\frac{\omega_{ \pm}\left(\ell_{ \pm}\right)}{\gamma_{12}}\right)
$$

where $p=+1$ for $F_{1}$-philic and $p=-1$ for $F_{2}$-philic substrates $S_{ \pm}$. This equation connects the macroscopic contact angle $\vartheta_{ \pm}$with the microscopic structure represented by the effective interface potential $\omega_{ \pm}(\ell)$ of $A$-films at substrate $S_{ \pm}$in macroscopic contact with bulk fluid $B$.

The next Sec. IIC describes an approximate calculation of the effective interface potentials $\omega_{ \pm}(\ell)$ for the setting of Fig. 1. The dependence of $\omega_{ \pm}\left(\ell_{ \pm} ; U\right)$ on the electrostatic potential difference $U$ between the electrodes, together with Eq. (6), then leads to the electrowetting equations derived in Sec. IID

However, already without explicit expressions for the effective interface potentials, one can draw an important conceptual conclusion from Eq. (6) : Electrowetting is not an electrocapillarity effect, since no $U$-dependent substrate-fluid interfacial tensions, which describe the contact of the substrate with one fluid, occur on the right-hand side. Instead, electrowetting is related to the depth of the effective interface potential, which describes the $U$-dependence of the microscopic fluid structure close to the substrate in the presence of two fluids.

\section{Density functional theory of wetting}

In order to obtain the effective interface potential $\omega_{ \pm}(\ell)$ of an $A$-film of thickness $\ell$ at substrate $S_{ \pm}$in Fig. 1 whose value for the equilibrium film thicknesses $\ell=\ell_{ \pm}$ is related to the contact angle $\vartheta_{ \pm}$via Eq. (6), one may represent the structure in Fig. 1 far above (for an $F_{1^{-}}$ philic substrate $S_{ \pm}$, see Fig. 2(a)) or below (for an $F_{2^{-}}$ philic substrate $S_{ \pm}$, see Fig. 2(b)) the three-phase contact region by the quasi-one-dimensional slab depicted 


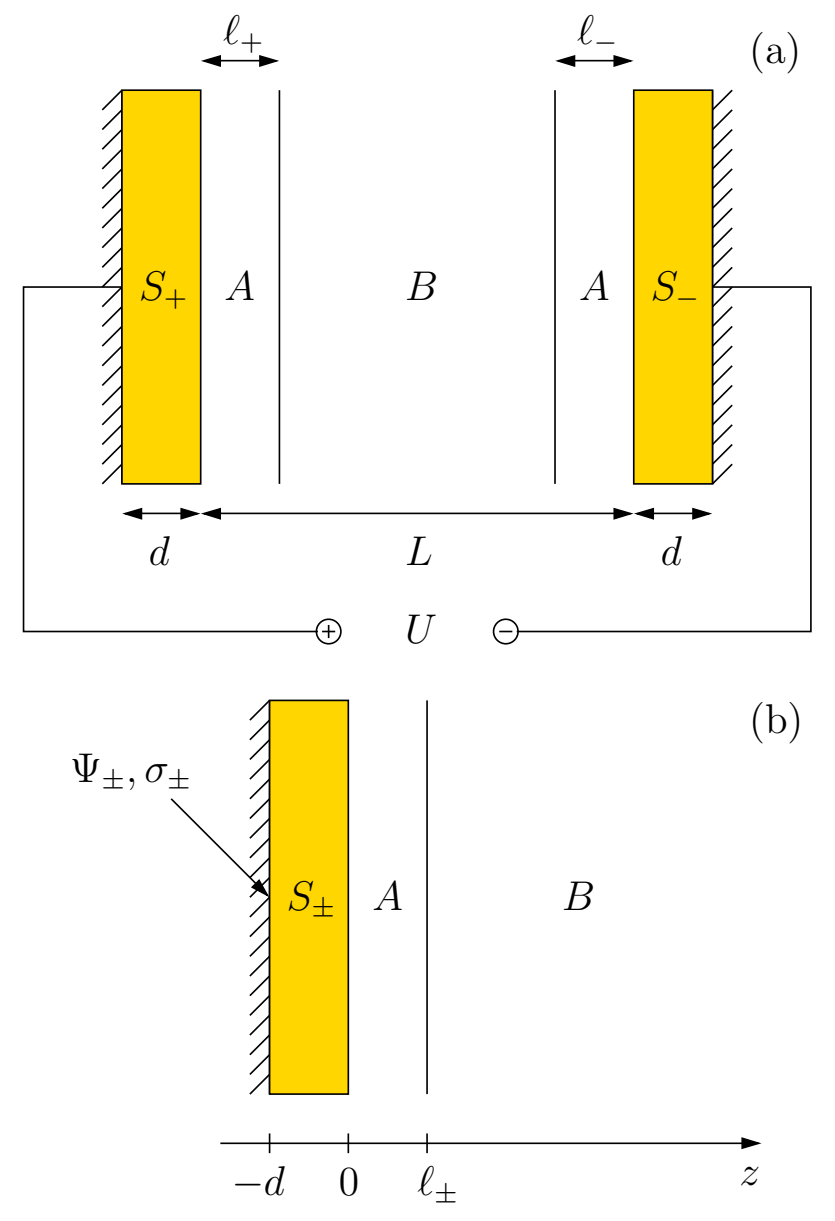

FIG. 3: (a) Far above (Fig. 2(a)) or below (Fig. 2(b)) the three-phase contact regions (see Fig. 1) the dielectric substrates $S_{+}$and $S_{-}$of thickness $d$ and distance $L$ are covered by films of the preferred fluid $A$ of microscopic thicknesses $\ell_{ \pm}$, which separate the substrates from the bulk of the less preferred fluid $B$. (b) Since the separation $L$ between the substrates $S_{+}$and $S_{-}$is typically the largest length scale, one can consider the limit $L \rightarrow \infty$, which renders the effective interface potential $\omega_{ \pm}(\ell)$ at substrate $S_{ \pm}$that of a semi-infinite system. A coordinate axis normal to each substrate is introduced with the origin $z=0$ at the surface and the fluids in the range $z>0$. The interface between the $A$-film and the bulk fluid $B$ is located at $z=\ell_{ \pm}$and the electrode is at position $z=-d$, where the electrostatic potential is $\Psi_{ \pm}$and the surface charge density is $\sigma_{ \pm}$.

in Fig. 3(a). The chemically identical substrates $S_{+}$and $S_{-}$, which comprise metal electrodes coated with dielectric layers of thickness $d$, are separated by a distance $L$ and covered with films of thicknesses $\ell_{+}$and $\ell_{-}$, respectively, of the preferred fluid $A$. Moreover, the electrodes are assumed to be ideally polarized, i.e., electrochemical reactions do not occur. Even under these conditions the film thicknesses $\ell_{+}$and $\ell_{-}$can differ, if unequal partitioning of ions at the film-bulk fluid interfaces takes place. This is expected to occur in general due to generic differences in solubility contrasts [36]. The macroscopic distance $L$ between the substrates is typically the largest length scale such that only the limit $L \rightarrow \infty$ is considered in the following. Hence the effective interface potentials $\omega_{+}(\ell)$ and $\omega_{-}(\ell)$ at the substrates $S_{+}$and $S_{-}$, respectively, are those of semi-infinite systems. For each substrate $S_{ \pm}$a coordinate axis in normal direction with the origin $z=0$ at the substrate surface and the fluid at $z>0$ is introduced (see Fig. B(b)). The interface between the $A$-film and the bulk of phase $B$ is located at position $z=\ell_{ \pm}$and the electrode is at $z=-d$, where the electrostatic potential is $\Psi_{ \pm}$and the surface charge density is $\sigma_{ \pm}$.

Considering the two bulk phases of fluids $F_{1}$ and $F_{2}$ outside the capacitor in Fig. 1 as particle reservoirs, one is naturally led to a grand-canonical description of the thermodynamic state. A starting point for the derivation of the effective interface potential $\omega_{ \pm}(\ell)$ is the grand potential functional per thermal energy $k_{B} T=1 / \beta$ and per area $\mathcal{A}$ of the electrode

$$
\begin{gathered}
\frac{\beta \Omega_{1}\left[\phi, \varrho_{ \pm}\right]}{\mathcal{A}}=\frac{\beta \Omega_{0}[\phi]}{\mathcal{A}}+\frac{\beta d D(0)^{2}}{2 \varepsilon_{\mathrm{vac}} \varepsilon_{S}}-\beta \Psi_{ \pm} D(0) \\
+\int_{0}^{\infty} \mathrm{d} z\left[\sum_{i= \pm} \varrho_{i}(z)\left(\ln \frac{\varrho_{i}(z)}{\zeta_{i}}-1+\beta V_{i}(\phi(z))\right)\right. \\
\left.+\frac{\beta D(z)^{2}}{2 \varepsilon_{\mathrm{vac}} \varepsilon(\phi(z))}\right]
\end{gathered}
$$

in terms of the solvent composition profile $\phi$ and the \pm ion number density profiles $\varrho_{ \pm}$. Here the permittivity $\varepsilon_{\mathrm{vac}}$ of the vacuum, the relative permittivity $\varepsilon_{S}$ of the substrate $S_{ \pm}$, and the fugacities $\zeta_{ \pm}$of \pm -ions are used. The density functional $\Omega_{0}[\phi]$ describes the grand potential of the pure, i.e., salt-free fluids. The electric displacement $D(z)$ is determined by Gauß's law and the boundary condition of global charge neutrality:

$$
D^{\prime}(z)=\sum_{i= \pm} q_{i} e \varrho_{i}(z), \quad D(\infty)=0 .
$$

Here $q_{ \pm}= \pm 1$ denotes the valency of \pm -ions and $e$ is the elementary charge. Since the substrate $S_{ \pm}$is free of ions the electrode surface charge density is given by $\sigma_{ \pm}=D\left(-d^{+}\right)=D\left(0^{-}\right)$(see Fig. B(b)). In the absence of specific adsorption of ions at the substrate surface, the electric displacement is continuous at $z=0$, i.e., $D\left(0^{-}\right)=D\left(0^{+}\right)=D(0)$, so that $\sigma_{ \pm}=D(0)$. The electric displacement $D(z)$ is related to the electrostatic potential $\psi(z)$ by $D(z)=-\varepsilon_{\mathrm{vac}} \varepsilon(\phi(z)) \psi^{\prime}(z)$. The electrostatic potential of the electrode is given by $\Psi_{ \pm}=\psi(-d)$ (see Fig. 3(b)). The second term on the right-hand side and the term in the last line of Eq. (77) account for the electrostatic field energy inside the substrate and the fluids, respectively, whereas the last term in the first line represents the internal energy of the voltage source sustaining the electrostatic potential $\psi(-d)=\Psi_{ \pm}$of the electrode (see Fig. 3(b)). The expressions $V_{ \pm}(\phi(z))$ and $\varepsilon(\phi(z))$ denote respectively the local solvation free energy of a \pm -ion 
and the relative permittivity at a position $z>0$ where the solvent composition is given by $\phi(z)$ [36]. Finally, the second line of Eq. (7) describes the grand potential of the ions, where the ion number densities are assumed to be sufficiently small such that ions interact with each other only via the electrostatic field.

The equilibrium bulk state $(\bar{\phi}, I)$ with the bulk solvent composition $\bar{\phi}$ and the bulk ionic strength $I$ minimizes the bulk grand potential density $\Omega_{b}(\bar{\phi}, I) / \mathcal{V}$, which can be derived from Eq. (7) by inserting uniform profiles $\phi(z)=\bar{\phi}$ and $\varrho_{ \pm}(z)=I$, omitting all surface terms, and noting $D=0$ in the bulk. The two immiscible fluids $A$ and $B$ at coexistence in Fig. 3(a) correspond to two equilibrium bulk states $\left(\bar{\phi}_{A}, I_{A}\right)$ and $\left(\bar{\phi}_{B}, I_{B}\right)$, respectively, with equal bulk grand potential density: $\Omega_{b}\left(\bar{\phi}_{A}, I_{A}\right) / \mathcal{V}=\Omega_{b}\left(\bar{\phi}_{B}, I_{B}\right) / \mathcal{V}$.

Since the present analysis is concerned with films of thicknesses $\ell_{ \pm}>\xi$ but not with interfacial structures on length scales less than $\xi$ it is natural to approximate the solvent composition profile $\phi$ in Eq. (7) within the so-called sharp-kink approximation [20]

$$
\phi_{\ell}(z):= \begin{cases}\bar{\phi}_{A}, & z<\ell \\ \bar{\phi}_{B}, & z>\ell\end{cases}
$$

Furthermore, in view of the small ionic strengths $I_{A}$ and $I_{B}$ to be considered here and for sufficiently small surface potential $|\psi(0)-\psi(\infty)|$, the term in the second line of Eq. (7) can be expanded up to quadratic order in the ion number density deviations $\Delta \varrho_{i}(z):=\varrho_{i}(z)-I_{\alpha}, i= \pm$, with $\alpha=A$ for $z<\ell$ and $\alpha=B$ for $z>\ell$ if $I_{\alpha}>0$. This leads to an approximate grand potential functional

$$
\begin{array}{r}
\frac{\beta \Omega\left(\ell,\left[\Delta \varrho_{ \pm}\right]\right)}{\mathcal{A}}=\frac{\beta \Omega_{0}\left[\phi_{\ell}\right]}{\mathcal{A}}+\frac{\beta d D(0)^{2}}{2 \varepsilon_{\mathrm{vac}} \varepsilon_{S}}-\beta \Psi_{ \pm} D(0) \\
+\int_{0}^{\ell} \mathrm{d} z\left[f_{A}\left(\Delta \varrho_{+}(z), \Delta \varrho_{-}(z)\right)+\frac{\beta D(z)^{2}}{2 \varepsilon_{\mathrm{vac}} \varepsilon_{A}}\right] \\
+\int_{\ell}^{\infty} \mathrm{d} z\left[f_{B}\left(\Delta \varrho_{+}(z), \Delta \varrho_{-}(z)\right)+\frac{\beta D(z)^{2}}{2 \varepsilon_{\mathrm{vac}} \varepsilon_{B}}\right]
\end{array}
$$

with

$$
\begin{aligned}
& f_{\alpha}\left(\Delta \varrho_{+}, \Delta \varrho_{+}\right)= \\
& \begin{cases}\sum_{i= \pm}\left[I_{\alpha}\left(\ln \frac{I_{\alpha}}{\zeta_{i}}-1+\beta V_{i}\left(\bar{\phi}_{\alpha}\right)\right)\right. \\
\left.\quad+\left(\ln \frac{I_{\alpha}}{\zeta_{i}}+\beta V_{i}\left(\bar{\phi}_{\alpha}\right)\right) \Delta \varrho_{i}+\frac{\Delta \varrho_{i}^{2}}{2 I_{\alpha}}\right], & I_{\alpha}>0 \\
0, & I_{\alpha}=0 .\end{cases}
\end{aligned}
$$

By minimizing $\beta \Omega\left(\ell,\left[\Delta \varrho_{ \pm}\right]\right) / \mathcal{A}$ in Eq. (10) with respect to the profiles $\Delta \varrho_{ \pm}$one obtains the equilibrium profiles $\Delta \varrho_{ \pm}^{(\ell)}$. Inserting $\Delta \varrho_{ \pm}^{(\ell)}$ into Eq. (10) and subtracting the bulk contribution $\beta \Omega_{b}\left(\bar{\phi}_{B}, I_{B}\right) / \mathcal{A}$ leads to the surface contribution to the grand potential [20]

$$
\Omega_{s}(\ell)=\frac{\Omega\left(\ell,\left[\Delta \varrho_{ \pm}^{(\ell)}\right]\right)-\Omega_{b}\left(\bar{\phi}_{B}, I_{B}\right)}{\mathcal{A}} .
$$

Finally, the effective interface potential at substrate $S_{ \pm}$ is given by $\omega_{ \pm}(\ell)=\Omega_{s}(\ell)-\Omega_{s}(\infty)$ [20], which, in the present context, can be written in the form

$$
\begin{aligned}
\omega_{ \pm}(\ell)= & \omega_{0}(\ell)+\frac{A(\ell)}{2}\left(\Psi_{ \pm}-\bar{\psi}_{A}\right)^{2} \\
& +B(\ell)\left(\Psi_{ \pm}-\bar{\psi}_{A}\right)+C(\ell) .
\end{aligned}
$$

Here $\omega_{0}(\ell)$ denotes the effective interface potential corresponding to the grand potential functional $\Omega_{0}[\phi]$ of the pure fluids, $\bar{\psi}_{\alpha}:=-\left(k_{B} T \ln \left(I_{\alpha} / \zeta_{+}\right)+V_{+}\left(\bar{\phi}_{\alpha}\right)\right) / e=$ $\left(k_{B} T \ln \left(I_{\alpha} / \zeta_{-}\right)+V_{-}\left(\bar{\phi}_{\alpha}\right)\right) / e$ is the Galvani potential of phase $\alpha \in\{A, B\}$, and

$$
\begin{aligned}
& A(\ell)=\frac{\varepsilon_{\mathrm{vac}}}{Q(\ell) \lambda_{B}} \frac{\lambda_{B}-\lambda_{A}}{\lambda_{S}+\lambda_{A}} \exp \left(-\frac{\lambda_{\ell}}{\lambda_{A}}\right) \\
& B(\ell)=-\frac{\varepsilon_{\mathrm{vac}}}{Q(\ell) \lambda_{B}}\left(\bar{\psi}_{A}-\bar{\psi}_{B}\right) \\
& C(\ell)=\frac{\varepsilon_{\mathrm{vac}}}{2 Q(\ell) \lambda_{B}} \frac{\lambda_{S}-\lambda_{A}}{\lambda_{B}+\lambda_{A}} \exp \left(-\frac{\lambda_{\ell}}{\lambda_{A}}\right)\left(\bar{\psi}_{A}-\bar{\psi}_{B}\right)^{2} \\
& Q(\ell)=\cosh \left(\frac{\lambda_{\ell}}{\lambda_{A}}\right)\left(1+\frac{\lambda_{S}}{\lambda_{B}}\right)+\sinh \left(\frac{\lambda_{\ell}}{\lambda_{A}}\right)\left(\frac{\lambda_{A}}{\lambda_{B}}+\frac{\lambda_{S}}{\lambda_{A}}\right)
\end{aligned}
$$

with the length scales $\lambda_{A}:=1 /\left(\kappa_{A} \varepsilon_{A}\right), \quad \lambda_{B}:=$ $1 /\left(\kappa_{B} \varepsilon_{B}\right), \lambda_{S}:=d / \varepsilon_{S}$, and $\lambda_{\ell}:=\ell / \varepsilon_{A}$, where $\kappa_{\alpha}^{2}:=$ $2 \beta e^{2} I_{\alpha} /\left(\varepsilon_{\mathrm{vac}} \varepsilon_{\alpha}\right)$ is the square of the inverse Debye length in the bulk of phase $\alpha \in\{A, B\}$. In addition, the electrode charge density can be written as

$$
\sigma_{ \pm}=D(0)=F\left(\ell_{ \pm}\right)\left(\Psi_{ \pm}-\bar{\psi}_{A}\right)-B\left(\ell_{ \pm}\right)
$$

with

$$
F(\ell)=\frac{\varepsilon_{\mathrm{vac}}}{Q(\ell)}\left(\frac{1}{\lambda_{A}} \sinh \left(\frac{\lambda_{\ell}}{\lambda_{A}}\right)+\frac{1}{\lambda_{B}} \cosh \left(\frac{\lambda_{\ell}}{\lambda_{A}}\right)\right)
$$

The Galvani potential difference (Donnan potential) between the phases $A$ and $B, \bar{\psi}_{A}-\bar{\psi}_{B}=\left(\left(V_{-}\left(\bar{\phi}_{A}\right)-\right.\right.$ $\left.\left.V_{+}\left(\bar{\phi}_{A}\right)\right)-\left(V_{-}\left(\bar{\phi}_{B}\right)-V_{+}\left(\bar{\phi}_{B}\right)\right)\right) /(2 e)$, can be inferred from the solubility of the \pm -ions in the solvents $A$ and $B$ [22, 37 39]. Moreover, $\Psi_{ \pm}-\bar{\phi}_{A}$ is determined by the potential difference $U=\Psi_{+}-\Psi_{-}$and the fact that no chemical reactions take place at the electrodes so that the total charge of both electrodes vanishes: $\sigma_{+}+\sigma_{-}=0$. Using Eq. (15) leads to

$$
\begin{aligned}
& \Psi_{+}-\bar{\psi}_{A}=\frac{F\left(\ell_{-}\right) U+B\left(\ell_{+}\right)+B\left(\ell_{-}\right)}{F\left(\ell_{+}\right)+F\left(\ell_{-}\right)} \\
& \Psi_{-}-\bar{\psi}_{A}=\frac{-F\left(\ell_{+}\right) U+B\left(\ell_{+}\right)+B\left(\ell_{-}\right)}{F\left(\ell_{+}\right)+F\left(\ell_{-}\right)}
\end{aligned}
$$

\section{Electrowetting equation}

The equilibrium film thicknesses $\ell_{+}$and $\ell_{-}$are both similar in magnitude $\left(\ell_{+} \approx \ell_{-}\right)$of the order of a few 
bulk correlation lengths $\xi$ away from wetting transitions. Hence, Eq. (17) leads to $\Psi_{ \pm}-\bar{\psi}_{A} \approx \pm U / 2+\left(B\left(\ell_{+}\right)+\right.$ $\left.B\left(\ell_{-}\right)\right) /\left(F\left(\ell_{+}\right)+F\left(\ell_{-}\right)\right)$, i.e., the $U$-dependent part of $\Psi_{ \pm}-\bar{\psi}_{A}$ is rather insensitive to a variation of $\ell_{+} \approx \ell_{-}$. Moreover, the film thicknesses $\ell_{+}$and $\ell_{-}$are typically much smaller than the Debye length $1 / \kappa_{A}$ in the $A$ film. Consequently, the leading $U$-dependent contribution $\sim U^{2}$ to the effective interface potential $\omega_{ \pm}(\ell)$ in Eq. (13), which decays exponentially with $\ell$ on the length scale of half of a Debye length, $1 /\left(2 \kappa_{A}\right)$ (see Eq. (14)), does not significantly shift the equilibrium film thickness $\ell_{ \pm}$but it merely lifts the depth $\omega_{ \pm}\left(\ell_{ \pm} ; U\right)$ of the effective interface potential. Therefore, in the following, the film thicknesses $\ell_{ \pm}$are considered as independent of the applied voltage $U$.

Hence, Eq. (6) can be written as [7]

$$
\cos \vartheta_{ \pm}(U)=\cos \vartheta_{ \pm}(0)+\eta_{ \pm}(U)
$$

with the electrowetting number

$$
\eta_{ \pm}(U):=p \frac{\omega_{ \pm}\left(\ell_{ \pm} ; U\right)-\omega_{ \pm}\left(\ell_{ \pm} ; 0\right)}{\gamma_{12}} .
$$

Inserting Eq. (17) into Eq. (13) leads to

$$
\begin{aligned}
& \eta_{ \pm}(U)=\frac{p}{\gamma_{12}}\left(\frac{A\left(\ell_{ \pm}\right) F\left(\ell_{\mp}\right)^{2}}{2\left(F\left(\ell_{+}\right)+F\left(\ell_{-}\right)\right)^{2}} U^{2}\right. \pm\left(\frac{A\left(\ell_{ \pm}\right) F\left(\ell_{\mp}\right)\left(B\left(\ell_{+}\right)+B\left(\ell_{-}\right)\right)}{\left(F\left(\ell_{+}\right)+F\left(\ell_{-}\right)\right)^{2}}\right. \\
&\left.\left.\quad+\frac{B\left(\ell_{ \pm}\right) F\left(\ell_{\mp}\right)}{F\left(\ell_{+}\right)+F\left(\ell_{-}\right)}\right) U\right) .
\end{aligned}
$$

This equation is expected to be valid for sufficiently small voltages $|U|$ such that the quadratic approximation Eq. (11) is applicable. The electrowetting number $\eta_{ \pm}(U)$ in Eq. (20) differs from those in the literature [7] in a number of aspects, as is discussed in the next section. The most obvious difference is the occurrence of a correction term $\sim U$, which vanishes exactly only if $B(\ell)=0$ due to a vanishing Donnan potential (Galvani potential difference) $\bar{\psi}_{A}-\bar{\psi}_{B}$. For $\bar{\psi}_{A}-\bar{\psi}_{B} \neq 0$, i.e., $B(\ell) \neq 0$, the electrowetting number $\eta_{ \pm}(U)$ in Eq. (20) is not minimal at and not symmetric with respect to $U=0$. However, for a sufficiently large voltage $|U|$ the subleading term $\sim U$ is dominated by the leading term $\sim U^{2}$.

Whereas the full expression for the electrowetting number $\eta_{ \pm}(U)$ in Eq. (20) depends on the five possibly largely different length scales $\lambda_{A}, \lambda_{B}, \lambda_{S}, \lambda_{\ell_{+}}$, and $\lambda_{\ell_{-}}$, the latter two, corresponding to the thicknesses of the $A$-films at the substrates $S_{+}$and $S_{-}$, respectively, are typically of similar magnitude: $\ell_{+} \approx \ell_{-}$, i.e., $\lambda_{\ell_{+}} \approx \lambda_{\ell_{-}}$. This case $\ell_{+}=\ell_{-}=: \ell$ is discussed in detail in the next section, for which the electrowetting number $\eta_{ \pm}(U)$ in Eq. (20) simplifies to

$$
\eta_{ \pm}(U)=\frac{p}{\gamma_{12}}\left(\frac{A(\ell)}{8} U^{2} \pm \frac{B(\ell)}{2}\left(\frac{A(\ell)}{F(\ell)}+1\right) U\right) .
$$

Moreover, the film thicknesses $\ell_{+}$and $\ell_{-}$are typically smaller than the Debye lengths $1 / \kappa_{A}$ and $1 / \kappa_{B}$ so that the limiting case $\lambda_{\ell} \ll \lambda_{A}, \lambda_{B}$ is considered throughout, within which

$$
\begin{aligned}
& A(\ell) \simeq \frac{\varepsilon_{\mathrm{vac}}}{Q(\ell)} \frac{\lambda_{B}-\lambda_{A}}{\lambda_{B}\left(\lambda_{S}+\lambda_{A}\right)} \\
& B(\ell)=-\frac{\varepsilon_{\mathrm{vac}}}{Q(\ell)} \frac{\bar{\psi}_{A}-\bar{\psi}_{B}}{\lambda_{B}} \\
& F(\ell) \simeq \frac{\varepsilon_{\mathrm{vac}}}{Q(\ell)}\left(\frac{\lambda_{\ell}}{\lambda_{A}^{2}}+\frac{1}{\lambda_{B}}\right) \\
& Q(\ell) \simeq 1+\frac{\lambda_{S}}{\lambda_{B}}+\frac{\lambda_{\ell} \lambda_{S}}{\lambda_{A}^{2}} .
\end{aligned}
$$

\section{DISCUSSION}

\section{A. Electrowetting and electrocapillarity}

Before discussing the electrowetting number in Eq. (21) obtained within the present density functional analysis, the traditional approach based on the assumption of electrowetting being an electrocapillarity effect [2, 3, [5, 7,19$]$ is repeated. Here only the classical method based on Lippmann's equation is presented. However, calculations using alternative methods, e.g., based on Maxwell's stress tensor [7, 40], suffer from the same misconceptions.

The starting point is Young's equation (3) but with the incorrect assumption $\Omega_{s, \pm \alpha}=\gamma_{ \pm \alpha}, \alpha \in\left\{F_{1}, F_{2}\right\}$. In order to obtain the $U$-dependence of the interfacial tension $\gamma_{ \pm \alpha}$ one considers a semi-infinite fluid $\alpha$ bound by a planar substrate $S_{ \pm}$. The interfacial tension $\gamma_{ \pm \alpha}$ changes upon changing the electrostatic potential $\psi_{ \pm \alpha}$ of substrate $S_{ \pm}$with respect to that of the bulk of phase $\alpha$ according to Lippmann's equation [1, 4]

$$
\frac{\partial \gamma_{ \pm \alpha}}{\partial \psi_{ \pm \alpha}}=-\sigma_{ \pm \alpha}
$$

where $\sigma_{ \pm \alpha}$ is the surface charge density of substrate $S_{ \pm}$ in contact with phase $\alpha$. Describing the $S_{ \pm-} \alpha$ interface by means of the potential-independent differential capacitance $C_{S \alpha}=\partial \sigma_{ \pm \alpha} / \partial \psi_{ \pm \alpha}$, which is assumed to not depend on $S_{ \pm}$for chemically identical substrates, and integrating twice with respect to the electrostatic substrate potential $\psi_{ \pm \alpha}$ using Lippmann's equation (26) leads to

$$
\gamma_{ \pm \alpha}\left(\psi_{ \pm \alpha}\right)=\gamma_{ \pm \alpha}(0)-\frac{C_{S \alpha}}{2} \psi_{ \pm \alpha}^{2} .
$$

Young's equation (3) in conjunction with the assumption $\Omega_{s, \pm \alpha}=\gamma_{ \pm \alpha}$ reads

$$
\begin{aligned}
\gamma_{ \pm 2}\left(\psi_{ \pm 2}\right) & =\gamma_{ \pm 2}(0)-\frac{C_{S 2}}{2} \psi_{ \pm 2}^{2} \\
& =\gamma_{ \pm 1}\left(\psi_{ \pm 1}\right)+\gamma_{12} \cos \vartheta_{ \pm} \\
& =\gamma_{ \pm 1}(0)-\frac{C_{S 1}}{2} \psi_{ \pm 1}^{2}+\gamma_{12} \cos \vartheta_{ \pm}
\end{aligned}
$$


Noting $\gamma_{ \pm 2}(0)-\gamma_{ \pm 1}(0)=\gamma_{12} \cos \vartheta_{ \pm}(0)$ leads to

$$
\cos \vartheta_{ \pm}-\cos \vartheta_{ \pm}(0)=\frac{C_{S 1}}{2 \gamma_{12}} \psi_{ \pm 1}^{2}-\frac{C_{S 2}}{2 \gamma_{12}} \psi_{ \pm 2}^{2} .
$$

Using $\sigma_{ \pm \alpha}=C_{S \alpha} \psi_{ \pm \alpha}$, one obtains the analog of Eq. (17) from $U=\psi_{+\alpha}-\psi_{-\alpha}$ and $\sigma_{+\alpha}+\sigma_{-\alpha}=0$ as $\psi_{ \pm \alpha}=$ $\pm U / 2$. This leads to the commonly used form of the electrowetting equation 2, 3, 5, $7-19$

$$
\cos \vartheta_{ \pm}(U)-\cos \vartheta_{ \pm}(0)=\frac{C_{S 1}-C_{S 2}}{8 \gamma_{12}} U^{2}=: \eta_{\mathrm{ec}}(U)
$$

with the differential capacitances $C_{S \alpha}$ being those of a substrate in macroscopic contact with only one fluid phase $\alpha$. These differential capacitances $C_{S \alpha}$ can typically be interpreted as those of a capacitor of capacitance $C_{S}=\varepsilon_{\mathrm{vac}} \varepsilon_{S} / d$, representing substrate $S_{ \pm}$, connected in series with a capacitor of capacitance $C_{\alpha}$, representing fluid $\alpha: 1 / C_{S \alpha}=1 / C_{S}+1 / C_{\alpha}$. If fluid $\alpha$ is an electrolyte solution the fluid capacitance is that of the electric double layer in a semi-infinite system, $C_{\alpha}=\varepsilon_{\mathrm{vac}} \kappa_{\alpha} \varepsilon_{\alpha}$, whereas for a non-conducting dielectric fluid $C_{\alpha}=\lim _{L \rightarrow \infty} \varepsilon_{\mathrm{vac}} \varepsilon_{\alpha} / L=0$. Using the length scales defined after Eq. (14), this leads to

$$
C_{S \alpha}= \begin{cases}\frac{\varepsilon_{\mathrm{vac}}}{\lambda_{S}+\lambda_{\alpha}}, & \alpha \text { electrolyte solution } \\ 0, & \alpha \text { non-conducting fluid. }\end{cases}
$$

Equations (30) and (31) represent the interpretation of electrowetting as an electrocapillarity effect [7]. However, the crucial misconception underlying this interpretation is to use the approximation $\Omega_{s, \pm \alpha}=\gamma_{ \pm \alpha}$ and hence the differential capacitance $C_{S \alpha}$, which corresponds to a semi-infinite system of a single phase $\alpha$ bound by substrate $S_{ \pm}$, instead of accounting for the actual fluid structure at the substrate. The interfacial structure, and therefore surface quantities such as the surface contribution to the grand potential as well as the differential capacitance, of substrate $S_{ \pm}$in macroscopic contact with the bulk fluid $B$ depend significantly on whether the preferred fluid $A$ is present or not because an $A$-film forms in between the substrate $S_{ \pm}$and the bulk fluid $B$ in the former case whereas it does not in the latter case. In contrast, these structural properties are naturally accounted for within the present density functional approach, which relates the contact angle to the effective interface potential (see Eq. (6)), a quantity which correctly describes the contact of a substrate with both fluids $A$ and $B$.

\section{B. Electrowetting on uncoated metal electrodes}

The early investigations of electrocapillarity by Lippmann [1] and Pellat [2, 3] have been performed for metal electrodes without any dielectric coating. At that time for some decades mercury electrodes became the experimental standard for investigations of the electric double layer 4]. Pure metal electrodes can be considered as substrates with thickness $d$ being the smallest length scale: $\lambda_{S} \ll \lambda_{\ell} \ll \lambda_{A}, \lambda_{B}$. Within this limit one obtains $Q(\ell) \simeq 1$ from Eq. (25) and subsequently from Eqs. (22)(24)

$$
\begin{aligned}
& A(\ell) \simeq \varepsilon_{\mathrm{vac}}\left(\frac{1}{\lambda_{A}}-\frac{1}{\lambda_{B}}\right) \\
& B(\ell) \simeq-\frac{\varepsilon_{\mathrm{vac}}}{\lambda_{B}}\left(\bar{\psi}_{A}-\bar{\psi}_{B}\right) \\
& F(\ell) \simeq \varepsilon_{\mathrm{vac}}\left(\frac{\lambda_{\ell}}{\lambda_{A}^{2}}+\frac{1}{\lambda_{B}}\right) .
\end{aligned}
$$

For the case $\lambda_{A} \ll \lambda_{B}$, which is typically the case for hydrophilic substrates, an aqueous electrolyte solution $F_{1}=A$ (i.e., $p=+1$ ), and an oil $F_{2}=B$, one obtains for the the electrowetting number Eq. (21)

$$
\begin{aligned}
\eta_{ \pm}(U) & \simeq \frac{\varepsilon_{\mathrm{vac}}}{8 \gamma_{12} \lambda_{A}} U^{2} \mp \frac{\varepsilon_{\mathrm{vac}}\left(\bar{\psi}_{A}-\bar{\psi}_{B}\right)}{2 \gamma_{12}\left(\lambda_{A}+\lambda_{\ell} \lambda_{B} / \lambda_{A}\right)} U \\
& \simeq \frac{\varepsilon_{\mathrm{vac}}}{8 \gamma_{12} \lambda_{A}} U^{2}, \text { for }|U| \gg 4\left|\bar{\psi}_{A}-\bar{\psi}_{B}\right| .
\end{aligned}
$$

Hence, if the voltage $|U|$ is much larger than the Donnan potential (Galvani potential difference) $\left|\bar{\psi}_{A}-\bar{\psi}_{B}\right|$, the electrowetting number $\eta_{ \pm}(U)$ agrees with that in Eq. (30), where $C_{S 1} \simeq \varepsilon_{\text {vac }} / \lambda_{A}, C_{S 2}=0$ due to Eq. (31).

Similarly, for the case $\lambda_{A} \gg \lambda_{B}$, which is typically the case for hydrophobic substrates, an oil $F_{2}=A$ (i.e., $p=-1$ ), and an aqueous electrolyte solution $F_{1}=B$, one obtains for the electrowetting number Eq. (21)

$$
\begin{aligned}
\eta_{ \pm}(U) & \simeq \frac{\varepsilon_{\mathrm{vac}}}{8 \gamma_{12} \lambda_{B}} U^{2} \pm \frac{\varepsilon_{\mathrm{vac}}\left(\bar{\psi}_{A}-\bar{\psi}_{B}\right)}{2 \gamma_{12} \lambda_{A}} U \\
& \simeq \frac{\varepsilon_{\mathrm{vac}}}{8 \gamma_{12} \lambda_{B}} U^{2}, \quad \text { for }|U| \gg 4\left|\bar{\psi}_{A}-\bar{\psi}_{B}\right|
\end{aligned}
$$

Again, if the voltage $|U|$ is much larger than the Donnan potential (Galvani potential difference) $\left|\bar{\psi}_{A}-\bar{\psi}_{B}\right|$, the electrowetting number $\eta_{ \pm}(U)$ again agrees with that in Eq. (30), where $C_{S 1} \simeq \varepsilon_{\mathrm{vac}} / \lambda_{B}, C_{S 2}=0$ due to Eq. (31).

Therefore, the present formalism (Eqs. (21)-(25) confirms the electrocapillarity-based form of the electrowetting number for the case of uncoated metal electrodes $\left(\eta_{ \pm}(U) \simeq \eta_{\mathrm{ec}}(U)\right)$, provided the voltage $|U|$ is sufficiently large as compared to the Donnan potential (Galvani potential difference) $\left|\bar{\psi}_{A}-\bar{\psi}_{B}\right|$. Interestingly, for uncoated metal electrodes it is irrelevant whether they are $F_{1}$-philic (hydrophilic) or $F_{2}$-philic (hydrophobic).

However, a small voltage $|U| \ll\left|\bar{\psi}_{A}-\bar{\psi}_{B}\right|$ or $\lambda_{A} \approx$ $\lambda_{B}$, e.g., for two immiscible electrolyte solutions, leads to electrowetting numbers $\eta_{ \pm}(U) \sim U$, in contrast to $\eta_{\mathrm{ec}}(U) \approx 0$ in Eq. (30) due to $C_{S 1} \approx C_{S 2}$ according to Eq. (31). Since these conditions are rather special, this scenario is not expected to be of practical relevance, but it might provide a test for the present approach. 


\section{Electrowetting of water on hydrophobic dielectrics in oil}

In the last few decades most electrowetting settings used electrodes coated with an isolating dielectric for technical advantage 9]. Almost all of these studies used drops of an aqueous electrolyte solution $F_{1}$ placed on a hydrophobic dielectric and an oil $F_{2}$ as the environmental fluid in order to achieve large contact angle ranges being covered by electrowetting [7]. Within the present notation this situation corresponds to $A=F_{2}$ (i.e., $p=-1$ ) and $B=F_{1}$. Since the thickness $\ell$ of the microscopic oil film on the substrates $S_{ \pm}$is typically smaller than the the Debye length $1 / \kappa_{B}$ of the dilute electrolyte solution $B=F_{1}$, which in turn is typically much smaller than the thickness $d$ of the dielectric substrates $S_{ \pm}$, one identifies the case $\lambda_{\ell} \ll \lambda_{B} \ll \lambda_{S} \ll \lambda_{A}$, where a (practically) ion-free oil $A=F_{2}\left(I_{A} \approx 0\right)$ is assumed. For this regime Eqs. (22)- 25) read

$$
\begin{aligned}
& Q(\ell) \simeq \frac{\lambda_{S}}{\lambda_{B}} \\
& A(\ell) \simeq-\frac{\varepsilon_{\mathrm{vac}}}{\lambda_{S}} \\
& B(\ell) \simeq-\frac{\varepsilon_{\mathrm{vac}}}{\lambda_{S}}\left(\bar{\psi}_{A}-\bar{\psi}_{B}\right) \\
& F(\ell) \simeq \frac{\varepsilon_{\mathrm{vac}}}{\lambda_{S}}
\end{aligned}
$$

and hence Eq. (21) is given by

$$
\begin{aligned}
\eta_{ \pm}(U) & \simeq \frac{\varepsilon_{\mathrm{vac}}}{8 \gamma_{12} \lambda_{S}} U^{2} \pm \frac{\varepsilon_{\mathrm{vac}}\left(\bar{\psi}_{A}-\bar{\psi}_{B}\right)}{2 \gamma_{12} \lambda_{A}} U \\
& \simeq \frac{\varepsilon_{\mathrm{vac}}}{8 \gamma_{12} \lambda_{S}} U^{2}, \text { for }|U| \gg 4 \frac{\lambda_{S}}{\lambda_{A}}\left|\bar{\psi}_{A}-\bar{\psi}_{B}\right|
\end{aligned}
$$

Since $\lambda_{S} / \lambda_{A} \ll 1$, the approximation in the second line of the previous equation almost always applies. It shows that the electrowetting number $\eta_{ \pm}(U)$ for water on a hydrophobic dielectric in oil is also in agreement with $\eta_{\mathrm{ec}}(U)$ in Eq. (30) with $C_{S 1} \simeq \varepsilon_{\mathrm{vac}} / \lambda_{S}, C_{S 2}=0$ due to Eq. (31).

\section{Electrowetting of water on hydrophilic dielectrics in oil}

Replacing the hydrophobic dielectric substrate of the previous Sec. IIIC by a hydrophilic one leads to the case $A=F_{1}$ (i.e., $p=+1$ ), $B=F_{2}$, and $\lambda_{\ell} \ll \lambda_{A} \ll \lambda_{S} \ll$ $\lambda_{B}$, where again a (practically) ion-free oil $B=F_{2}\left(I_{B} \approx\right.$
$0)$ is assumed. For this regime Eqs. (22)- (25) read

$$
\begin{aligned}
& Q(\ell) \simeq 1+\frac{\lambda_{\ell} \lambda_{S}}{\lambda_{A}^{2}}=1+\frac{\varepsilon_{A}}{\varepsilon_{S}} \kappa_{A} \ell \kappa_{A} d \\
& A(\ell) \simeq \frac{\varepsilon_{\mathrm{vac}}}{Q(\ell)} \frac{1}{\lambda_{S}} \\
& B(\ell)=-\frac{\varepsilon_{\mathrm{vac}}}{Q(\ell)} \frac{\bar{\psi}_{A}-\bar{\psi}_{B}}{\lambda_{B}} \\
& F(\ell) \simeq \frac{\varepsilon_{\mathrm{vac}}}{Q(\ell)}\left(\frac{\lambda_{\ell}}{\lambda_{A}^{2}}+\frac{1}{\lambda_{B}}\right),
\end{aligned}
$$

which leads to Eq. (21) of the form

$$
\begin{aligned}
\eta_{ \pm}(U) \simeq \frac{1}{Q(\ell)}( & \frac{\varepsilon_{\mathrm{vac}}}{8 \gamma_{12} \lambda_{S}} U^{2} \mp \frac{\varepsilon_{\mathrm{vac}}\left(\bar{\psi}_{A}-\bar{\psi}_{B}\right)}{2 \gamma_{12} \lambda_{B}} \\
& \left.\times\left(\frac{1}{\lambda_{S}\left(\lambda_{\ell} / \lambda_{A}^{2}+1 / \lambda_{B}\right)}+1\right) U\right) \\
\simeq & \frac{1}{Q(\ell)} \frac{\varepsilon_{\mathrm{vac}}}{8 \gamma_{12} \lambda_{S}} U^{2}, \text { for }|U| \gg 4\left|\bar{\psi}_{A}-\bar{\psi}_{B}\right|
\end{aligned}
$$

Within the electrocapillarity approach one again expects, as in the previous Sec. IIIC, an electrowetting number $\eta_{\mathrm{ec}}(U)=\varepsilon_{\mathrm{vac}} U^{2} /\left(8 \gamma_{12} \lambda_{S}\right)$ (see Eqs. (30) and (31) ). However, the electrowetting number $\eta_{ \pm}(U)$ within the present density functional approach in Eq. (46), for sufficiently large voltage $|U| \gg 4\left|\bar{\psi}_{A}-\bar{\psi}_{B}\right|$, is actually smaller than $\eta_{\mathrm{ec}}(U)$ by a factor $1 / Q(\ell): \eta_{ \pm}(U) \simeq$ $\eta_{\mathrm{ec}}(U) / Q(\ell)$.

It is apparent from Eq. (42) that $Q(\ell)$ is not necessarily close to unity, because the typically small value $\kappa_{A} \ell \ll 1$ is multiplied with the typically large value $\kappa_{A} d \varepsilon_{A} / \varepsilon_{S} \gg$ 1. Assuming typical values of, e.g., dielectric layers of thicknesses $d=1 \mu \mathrm{m}$ and dielectric constant $\varepsilon_{S}=2$, a Debye length $1 / \kappa_{A}=10 \mathrm{~nm}$ in the aqueous $\left(\varepsilon_{A}=80\right)$ electrolyte solution $F_{1}=A$, and thicknesses $\ell=1 \mathrm{~nm}$ of the electrolyte films on the substrates, Eq. (42) leads to $Q(\ell) \approx 400$. Hence, for this example of electrowetting on a hydrophilic dielectric, the analysis leads to electrowetting numbers $\eta_{ \pm}(U)$ which are more than two orders of magnitude smaller than expected within the electrocapillarity approach: $\eta_{ \pm}(U) \approx 0.0025 \eta_{\mathrm{ec}}(U) \ll \eta_{\mathrm{ec}}(U)$.

It appears as if no experimental studies of electrowetting on hydrophilic substrates have been reported so far. This is remarkable since the preparation of hydrophilic substrates is standard in surface science.

\section{E. Electrowetting of immiscible electrolyte solutions on dielectrics}

Whereas the previous two Secs. IIIC and IIID considered an electrolyte solution and an oil, here the case of two immiscible electrolyte solutions is discussed. This situation is characterized by $\lambda_{\ell} \ll \lambda_{A}, \lambda_{B} \ll \lambda_{S}$, which 
leads to Eqs. (22)-25) of the form

$$
\begin{aligned}
& Q(\ell) \simeq \frac{\lambda_{S}}{\lambda_{A}}\left(\frac{\lambda_{A}}{\lambda_{B}}+\frac{\lambda_{\ell}}{\lambda_{A}}\right)=\frac{\varepsilon_{B}}{\varepsilon_{S}} \kappa_{B} d+\frac{\varepsilon_{A}}{\varepsilon_{S}} \kappa_{A} \ell \kappa_{A} d \\
& A(\ell) \simeq \frac{\varepsilon_{\mathrm{vac}}}{Q(\ell)} \frac{\lambda_{B}-\lambda_{A}}{\lambda_{S} \lambda_{B}} \\
& B(\ell)=-\frac{\varepsilon_{\mathrm{vac}}}{Q(\ell)} \frac{\bar{\psi}_{A}-\bar{\psi}_{B}}{\lambda_{B}} \\
& F(\ell) \simeq \frac{\varepsilon_{\mathrm{vac}}}{Q(\ell)}\left(\frac{\lambda_{\ell}}{\lambda_{A}^{2}}+\frac{1}{\lambda_{B}}\right)
\end{aligned}
$$

If electrolyte solutions $F_{1}$ and $F_{2}$ are defined by $\lambda_{F_{1}} \leq$ $\lambda_{F_{2}}$, i.e., $\varepsilon_{F_{1}} I_{F_{1}} \geq \varepsilon_{F_{2}} I_{F_{2}}$, the following three cases have to be distinguished: (i) $A=F_{1}$ (i.e., $p=+1$ ) and $B=F_{2}$ with $\lambda_{A} \ll \lambda_{B}$, (ii) $A=F_{2}$ (i.e., $p=-1$ ) and $B=F_{1}$ with $\lambda_{A} \gg \lambda_{B}$, and (iii) $\lambda_{A} \approx \lambda_{B}$.

Case (i) leads to the electrowetting number Eq. (21)

$$
\begin{aligned}
\eta_{ \pm}(U) & \simeq \frac{1}{Q(\ell)}\left(\frac{\varepsilon_{\mathrm{vac}}}{8 \gamma_{12} \lambda_{S}} U^{2} \mp \frac{\varepsilon_{\mathrm{vac}}\left(\bar{\psi}_{A}-\bar{\psi}_{B}\right)}{2 \gamma_{12} \lambda_{B}} U\right) \\
& \simeq \frac{1}{Q(\ell)} \frac{\varepsilon_{\mathrm{vac}}}{8 \gamma_{12} \lambda_{S}} U^{2}, \quad \text { for }|U| \gg 4 \frac{\lambda_{S}}{\lambda_{B}}\left|\bar{\psi}_{A}-\bar{\psi}_{B}\right| .
\end{aligned}
$$

Hence $\eta_{ \pm}(U) \simeq \eta_{\mathrm{ec}} / Q(\ell)$, where, however, the depression factor $1 / Q(\ell)$ here is typically much smaller than that of the previous Sec. IIID because typically $\varepsilon_{B} \kappa_{B} d / \varepsilon_{S} \gg 1$ (see Eqs. (42) and (47)).

The electrowetting number of case (ii) is given by

$$
\begin{aligned}
\eta_{ \pm}(U) & \simeq \frac{\lambda_{A}}{\lambda_{S}}\left(\frac{\varepsilon_{\mathrm{vac}}}{8 \gamma_{12} \lambda_{S}} U^{2} \pm \frac{\varepsilon_{\mathrm{vac}}\left(\bar{\psi}_{A}-\bar{\psi}_{B}\right)}{2 \gamma_{12} \lambda_{A}} U\right) \\
& \simeq \frac{\lambda_{A}}{\lambda_{S}} \frac{\varepsilon_{\mathrm{vac}}}{8 \gamma_{12} \lambda_{S}} U^{2}, \quad \text { for }|U| \gg 4 \frac{\lambda_{S}}{\lambda_{A}}\left|\bar{\psi}_{A}-\bar{\psi}_{B}\right| .
\end{aligned}
$$

This expression bears some resemblance to Eq. (41) except of the typically very small prefactor $\lambda_{A} / \lambda_{S} \ll 1$ here.

Therefore, electrowetting is also expected to be strongly suppressed for two immiscible electrolyte solutions with $\varepsilon_{F_{1}} I_{F_{1}} \not \approx \varepsilon_{F_{2}} I_{F_{2}}$, a condition which is typically fulfilled.

For completeness the rather special case (iii) is mentioned, for which the electrowetting number reads

$$
\eta_{ \pm}(U) \simeq \mp \frac{p \varepsilon_{\mathrm{vac}}\left(\bar{\psi}_{A}-\bar{\psi}_{B}\right)}{2 \gamma_{12} \lambda_{S}} U .
$$

\section{CONCLUSIONS AND SUMMARY}

Electrowetting is studied in the present work by analyzing the capillary rise of a fluid in the environment of another fluid, where at least one of the two fluids is an electrolyte solution, for Pellat's setup [2, 3] (Fig. 10) within the density functional theory of wetting. Here, the widely ignored possibility of the formation of films of microscopic thickness on the substrates is taken into account (Fig. 2). Considering the quasi-one-dimensional situation far away from the three-phase contact region (Fig. [3(a)) allows to transparently derive the electrowetting equation (18).

The derivation shows that electrowetting is a consequence not of the voltage-dependence of the substratefluid interfacial tensions, i.e., electrowetting is not an electrocapillarity effect, but of the voltage-dependence of the depth of the effective interface potential. The traditional electrocapillarity approach to electrowetting is shown to be compromised by the reliance on the incorrect assumption that the surface structure of a fluid does not change upon bringing the system into contact with another fluid phase.

The present analysis of Pellat's setup for electrowetting studies leads to effectively four length scales corresponding to the Debye lengths in both fluids, the thickness of the substrates, and the film thicknesses, the latter being assumed to be approximately equal here, which serve to classify various relevant experimental situations, e.g., uncoated metal electrodes, hydrophilic or hydrophobic dielectric substrates, or fluids comprising water+oil systems or immiscible electrolyte solutions. The full dependence of the electrowetting number on these length scales is derived here (Eq. (20)), which can be used for an actual experimental system. By considering limiting cases of general interest it is found that for uncoated metal electrodes (Eqs. (35) and (36)) and wetting of hydrophobic dielectric substrates by water in an oil environment (Eq. (41)) the electrowetting number within the present density functional approach agrees with that within the electrocapillarity picture as well as with numerous experimental studies. However, a significantly reduced tendency of electrowetting is predicted here as compared to the predictions within the electrocapillarity approach for electrowetting on hydrophilic dielectric substrates (Eq. (46)) or situations with both fluids being immiscible electrolyte solutions (Eqs. (51)-(53)). Due to a lack of experimental data, verification of these predictions is an open issue.

One can conclude that it is a matter of fortune that the traditional electrocapillarity approach to electrowetting (Eqs. (30) and (310) leads to an electrowetting equation, which, although derived by means of physically questionable arguments, turns out to be yet a precise approximation for many practical cases. However, the present study highlights conditions for which significant deviations from the electrocapillarity picture are expected to be experimentally detectable. The density functional theory of electrowetting presented here is suggested to be considered as an approach to fundamentally understand as well as to reliably quantify the phenomenon of electrowetting. 
[1] G. Lippmann, Ann. Chim. Phys. 5, 494 (1875).

[2] M.H. Pellat, C. R. Hebd. Séances Acad. Sci. 119, 675 (1894).

[3] M.H. Pellat, C. R. Hebd. Séances Acad. Sci. 121, 938 (1895).

[4] D.C. Grahame, Chem. Rev. 41, 441 (1947).

[5] M.J. Sparnaay, Surf. Sci. 1, 213 (1964).

[6] F.J. Holly, J. Colloid Interface Sci. 61, 435 (1977).

[7] F. Mugele and J.-C. Baret, J. Phys.: Condens. Matter 17, R705 (2005).

[8] J.A.M. Sondag-Huethorst and L.G.J. Fokkink, Langmuir 8, 2560 (1992)

[9] B. Berge, C. R. Acad. Sci. II 317, 157 (1993).

[10] M. Vallet, B. Berge, and L. Vovelle, Polymer 37, 2465 (1996).

[11] W.J.J. Welters and L.G.J. Fokkink, Langmuir 14, 1535 (1998).

[12] T.D. Blake, A. Clarke, and E.H. Stattersfield, Langmuir 16, 2928 (2000).

[13] C. Decamps and J. De Coninck, Langmuir 16, 10150 (2000).

[14] A. Quinn, R. Sedev, J. Ralston, J. Phys. Chem. B 107, 1163 (2003).

[15] T.B. Jones, K.-L. Wang, and D.-J. Yao, Langmuir 20, 2813 (2004).

[16] A. Quinn, R. Sedev, J. Ralston, J. Phys. Chem. B 109, $6268(2005)$

[17] D. Klarman and D. Andelman, Langmuir 27, 6031 (2011).

[18] R. Sedev, Eur. Phys. J. Special Topics 197, 307 (2011).

[19] C.D. Daub, D. Bratko, and A. Luzar, Top. Curr. Chem. 307, 155 (2012).

[20] S. Dietrich, in Phase transitions and critical phenomena, Vol. 12, edited by C. Domb and J.L. Lebowitz (Academic, London, 1988), p. 1.

[21] R.J. Hunter, Zeta potential in colloid science (Academic Press, London, 1981).

[22] J. Lyklema, Fundamentals of interface and colloid sci- ence, Vol. I (Academic Press, London, 1991).

[23] N.V. Churaev and Z.M. Zorin, Adv. Colloid Interface Sci. 40, 109 (1992).

[24] J. Lyklema, Fundamentals of interface and colloid science, Vol. II (Academic Press, London, 1995).

[25] A.W. Adamson and A.P. Gast, Physical chemistry of surfaces (Wiley, New York, 1997).

[26] R.J. Hunter, Foundations of colloid science (Oxford University Press, 2001).

[27] N.V. Churaev, Adv. Colloid Interface Sci. 103, 197 (2003).

[28] R. Digilov, Langmuir 16, 6719 (2000).

[29] C. Quilliet and B. Berge, Curr. Opin. Colloid Interface Sci. 6, 34 (2001).

[30] N.A. Denesyuk and J.-P. Hansen, Europhys. Lett. 63, 261 (2003).

[31] N.A. Denesyuk and J.-P. Hansen, J. Chem. Phys. 121, 3613 (2004).

[32] I. Ibagon, M. Bier, and S. Dietrich, J. Chem. Phys. 138, 214703 (2013).

[33] J.S. Rowlinson and B. Widom, Molecular Theory of Capillarity (Dover, Mineola, 2002).

[34] P.-G. de Gennes, F. Brochard-Wyart, and D. Queré, Capillarity and Wetting Phenomena (Springer, New York, 2004).

[35] L.D. Landau and E.M. Lifshitz, Fluid mechanics (Volume 6 of Course of Theoretical Physics) (Elsevier, Amsterdam, 2005).

[36] M. Bier, A. Gambassi, and S. Dietrich, J. Chem. Phys. 137, 034504 (2012).

[37] H.D. Inerowicz, W. Li, and I. Persson, J. Chem. Soc. Faraday Trans. 90, 2223 (1994).

[38] C. Kalidas, G. Hefter, and Y. Marcus, Chem. Rev. 100, 819 (2000).

[39] Y. Marcus, Chem. Rev. 107, 3880 (2007).

[40] K.H. Kang, Langmuir 18, 10318 (2002). 